\title{
Some refinements of a selection theorem with 0-dimensional domain
}

\author{
by
}

E. Michael (Seattle, Wash.)

\begin{abstract}
The following known selection theorem is sharpened, primarily, by weakening the hypothesis that all the sets $\varphi(x)$ are closed in $Y$ : Let $X$ be paracompact with $\operatorname{dim} X=0$, let $Y$ be completely metrizable and let $\varphi: X \rightarrow \mathcal{F}(Y)$ be l.s.c. Then $\varphi$ has a selection.
\end{abstract}

1. Introduction. The purpose of this note is to sharpen the following selection theorem, primarily by weakening the assumption that the sets $\varphi(x)$ are all closed in the complete metric space $Y$. Our study grew out of some valuable conversations with Roman Pol while he was a visitor at the University of Washington, and Corollary 1.6 answers a question which he asked.

Theorem $1.1\left[\mathrm{M}_{2}\right.$, Theorem 2]. Let $X$ be paracompact with $\operatorname{dim} X=0$, let $Y$ be completely metrizable, and let $\varphi: X \rightarrow \mathcal{F}(Y)$ be l.s.c. Then $\varphi$ has a selection.

In the above result, $\mathcal{F}(Y)$ denotes $\left\{E \in 2^{Y}: E\right.$ closed in $\left.Y\right\}$, where $2^{Y}=\{E \subset Y: E \neq \emptyset\}$. A function $\varphi: X \rightarrow 2^{Y}$ is l.s.c. (= lower semicontinuous) if $\{x \in X: \varphi(x) \cap V \neq \emptyset\}$ is open in $X$ for every open $V$ in $Y$, and a selection for $\varphi$ is a continuous $f: X \rightarrow Y$ such that $f(x) \in \varphi(x)$ for every $x \in X$.

The requirement in Theorem 1.1 that each $\varphi(x)$ be closed in $Y$ cannot be omitted (see $\left[\mathrm{M}_{6}\right.$, Example 9.1]). Before examining how it can be relaxed, we pause to record a generalization of Theorem 1.1 in a different direction which will form the basis for our subsequent results. If $A \subset X$ is closed, we say that $\varphi: X \rightarrow 2^{Y}$ has the $S E P$ (= selection extension property) at $A$ if every selection for $\varphi \mid A$ extends to a selection for $\varphi$; if $\varphi$ has the SEP at every closed $A \subset X$, then we simply say that $\varphi$ has the $S E P$ (see $\left[\mathrm{M}_{6}, \mathrm{p} .1\right]$ ).

The following result reduces to Theorem 1.1 when $A=\emptyset$. 
Theorem $1.2\left[\mathrm{M}_{4}\right.$, Theorem 1.2]. Let $X$ be paracompact, $Y$ completely metrizable, $A \subset X$ closed with $\operatorname{dim}_{X} X \backslash A=0\left({ }^{1}\right)$, and $\varphi: X \rightarrow \mathcal{F}(Y)$ l.s.c. Then $\varphi$ has the SEP at A.

We now turn to refinements of Theorem 1.2 which do not require that every $\varphi(x)$ be closed in $Y$. The simplest of these is the following result, which reduces to Theorem 1.2 when $C=\emptyset$.

Theorem $1.3\left[\mathrm{M}_{6}\right.$, Theorem 1.3]. Let $X, Y$ and $A \subset X$ be as in Theorem 1.2. Let $C \subset X$ be countable, and let $\varphi: X \rightarrow 2^{Y}$ be l.s.c. with $\varphi(x) \in \mathcal{F}(Y)$ for $x \notin C$. Then $\varphi$ has the SEP at $A$.

It was observed in $\left[\mathrm{M}_{6}\right.$, Section 8] that Theorem 1.3 remains valid if $C$ is only assumed to be a countable union of closed, discrete subsets of $X$. Proceeding further in this direction, we obtain the following generalization of Theorem 1.3. We say that $\varphi: X \rightarrow 2^{Y}$ has the SNEP (= selection neighborhood extension property) at a closed set $A \subset X$ if every selection for $\varphi \mid A$ extends to a selection for $\varphi \mid U$ for some open $U \supset A$; if $\varphi$ has the SNEP at every closed (resp. singleton) $A \subset X$, then we say that $\varphi$ has the $S N E P$ (resp. pointwise SNEP).

Theorem 1.4. Let $X, Y$ and $A \subset X$ be as in Theorem 1.2. Let $C=$ $\bigcup_{n} C_{n}$ with each $C_{n}$ closed in $X$, and let $\varphi: X \rightarrow 2^{Y}$ be l.s.c. with $\varphi(x) \in$ $\mathcal{F}(Y)$ for $x \notin C$ and with $\varphi \mid C_{n}$ having the SNEP for all $n$. Then $\varphi$ has the SEP at $A$.

Remark. Theorem 1.4 is of some interest even when $C=X$. In that case, $Y$ need only be metrizable (rather than completely metrizable), since we can then simply replace $Y$ by a completely metrizable space containing it.

We now come to the principal results of this paper. Theorem 1.5 sharpens Theorem 1.4 in a new direction, under the mild restriction that $A$ and the $C_{n}$ 's are $G_{\delta}$-subsets of $X\left({ }^{2}\right)$. We adopt the notation that, if $\varphi: X \rightarrow 2^{Y}$, then $\bar{\varphi}: X \rightarrow \mathcal{F}(Y)$ is defined by $\bar{\varphi}(x)=\overline{\varphi(x)}$. As observed in $\left[\mathrm{M}_{1}\right.$, Proposition 2.3], $\bar{\varphi}$ is l.s.c. if and only $\varphi$ is l.s.c.

Theorem 1.5. Let $X, Y, A \subset X$ and $C=\bigcup_{n} C_{n}$ be as in Theorem 1.4, and assume that $A$ and the $C_{n}$ 's are $G_{\delta}$-subsets of $X$. Let $Z$ be $a G_{\delta}$ in $Y$, and let $\varphi: X \rightarrow 2^{Y}$ be l.s.c. with $\varphi(x) \in \mathcal{F}(Z)$ for $x \notin C$, with $\bar{\varphi}(x) \cap Z$ dense in $\bar{\varphi}(x)$ for $x \in C\left({ }^{3}\right)$, and with $\varphi \mid C_{n}$ having the pointwise SNEP for all $n$. Then $\varphi$ has the SEP at $A$.

$\left({ }^{1}\right)$ This means that $X \backslash A$ is non-empty and $\operatorname{dim} S \leq 0$ for all closed (in $\left.X\right) S \subset X \backslash A$. If $X$ is metrizable (more generally, if $A$ is a $G_{\delta}$ in $X$ ), this is equivalent to $\operatorname{dim} X \backslash A=0$.

$\left({ }^{2}\right)$ Examples 6.1 and 6.2 show that this restriction cannot omitted.

$\left({ }^{3}\right)$ Example 6.3 shows that this assumption, which is automatically satisfied when $Z=Y$, cannot be omitted. 
While the statement of Theorem 1.5 is somewhat involved, it has the following easily stated corollary which answers a question raised by R. Pol.

Corollary 1.6. Let $X$ be metrizable with $\operatorname{dim} X=0$, let $Y$ be completely metrizable, $Z \subset Y$ a dense $G_{\delta}, C \subset X$ countable, and $\varphi: X \rightarrow 2^{Y}$ with $\varphi(x)=Z$ for $x \notin C$ and with $\bar{\varphi}(x)=Y$ all $x \in X$. Then $\varphi$ has the SEP.

We conclude this introduction with one more result. It was proved in $\left[\mathrm{M}_{7}\right.$, Theorem 1.2(b)] that Theorem 1.2 remains valid if the assumption that $\varphi(x)$ is closed in $Y$ for every $x \in X$ is weakened to assuming only that there exists a $G_{\delta}$-subset $Z$ of $X \times Y$ such that $\varphi(x)$ is relatively closed in $Z(x)$ for every $x \in X\left({ }^{4}\right)$. Similarly, Theorem 1.5 can be generalized as follows.

Theorem 1.7. Let $X, Y, A \subset X$ and $C=\bigcup_{n} C_{n}$ be as in Theorem 1.5. Let $Z$ be $a G_{\delta}$ in $X \times Y$, and let $\varphi: X \rightarrow 2^{Y}$ be l.s.c. with $\varphi(x) \in \mathcal{F}(Z(x))$ for $x \notin C$, with $\bar{\varphi}(x) \cap Z(x)$ dense in $\bar{\varphi}(x)$ for $x \in C$, and with $\varphi \mid C_{n}$ having the pointwise SNEP for all $n$. Then $\varphi$ has the SEP at $A$.

After establishing some lemmas in Section 2, Theorem 1.4 will be proved in Section 3, Theorem 1.5 in Section 4, and Theorem 1.7 in Section 5. Section 6 is devoted to examples.

2. Some lemmas. Lemmas 2.1 and 2.2 are simple observations, stated without proof. Lemma 2.3 will be applied in the proof of Theorem 1.4, and Lemma 2.5 and Corollary 2.6 in the proof of Theorem 1.5.

LEMMA 2.1. If $\varphi: X \rightarrow 2^{Y}$ has the pointwise SNEP, so does $\varphi \mid E$ for every $E \subset X$.

Lemma 2.2. Let $\varphi: X \rightarrow 2^{Y}$, let $W \subset X \times Y$ be open with $\varphi(x) \cap W(x)$ $\neq \emptyset$ for all $x \in X$, and define $\alpha: X \rightarrow 2^{Y}$ by $\alpha(x)=\varphi(x) \cap W(x)$. If $\varphi$ is l.s.c. or has the $S N E P$ at $A \subset X$, then $\alpha$ has the same property.

Lemma 2.3. Let $X$ be paracompact, $A \subset X$ closed with $\operatorname{dim}_{X} X \backslash A=0$, and let $\varphi: X \rightarrow 2^{Y}$ have the SNEP at $A$ and at every $x \in X \backslash A$. Then $\varphi$ has the SEP at $A$.

Proof. Let $g$ be a selection for $\varphi \mid A$. We must extend $g$ to a selection for $\varphi$.

Case 1: $\operatorname{dim} X=0$. Extend $g$ to a selection $h$ for $\varphi \mid U$ for some open $U \supset A$. Choose an open cover $\left(U_{\lambda}\right)$ of $X \backslash A$ such that $\varphi \mid U_{\lambda}$ has a selection $f_{\lambda}$ for each $\lambda$. The open cover $\{U\} \cup\left(U_{\lambda}\right)$ of $X$ has a disjoint open refinement $\left\{U^{\prime}\right\} \cup\left(U_{\lambda}^{\prime}\right)$. Define $f: X \rightarrow Y$ by $f\left|U^{\prime}=h\right| U^{\prime}$ and $f\left|U_{\lambda}^{\prime}=f_{\lambda}\right| U_{\lambda}^{\prime}$. This $f$ is a selection for $\varphi$ which extends $g$.

$\left({ }^{4}\right)$ For $E \subset X \times Y$ and $x \in X$, we define $E(x)=\{y \in Y:(x, y) \in E\}$. 
Case 2: $\operatorname{dim}_{X} X \backslash A=0$. As in Case 1, extend $g$ to selection $h$ for $\varphi \mid U$ for some open $U \supset A$. Pick $V$ open in $X$ with $A \subset V \subset \bar{V} \subset U$, and let $X^{*}=X \backslash V$ and $A^{*}=\bar{V} \backslash V$. Now $X^{*}$ is closed in $X$ and $X^{*} \subset X \backslash A$, so $\operatorname{dim} X^{*} \leq 0$. By Case 1 applied to $X^{*}$ and $A^{*}$, the selection $h \mid A^{*}$ for $\varphi \mid A^{*}$ extends to a selection $k$ for $\varphi \mid X^{*}$. Define $f: X \rightarrow Y$ by $f|\bar{V}=h| \bar{V}$ and $f \mid X^{*}=k$. This $f$ is a selection for $\varphi$ extending $g$.

Corollary 2.4. If $X$ is paracompact with $\operatorname{dim} X=0$, then $\varphi: X \rightarrow 2^{Y}$ has the (pointwise) SEP if and only if it has the (pointwise) SNEP.

Proof. This follows immediately from the special case of Lemma 2.3 where $\operatorname{dim} X=0$.

Lemma 2.5. Let $X$ be paracompact, $A \subset X$ a closed $G_{\delta}$ with $\operatorname{dim}_{X} X \backslash A$ $=0, Y$ metrizable, and let $\varphi: X \backslash A \rightarrow 2^{Y}$ have the pointwise SNEP. Suppose that $g: A \rightarrow Y$ and that $g$ extends to a continuous $f: X \rightarrow Y$ such that $f(x) \in \bar{\varphi}(x)$ for $x \in X \backslash A$. Then $g$ extends to a continuous $h: X \rightarrow Y$ such that $h(x) \in \varphi(x)$ for $x \in X \backslash A$.

Proof. Since $A$ is a closed $G_{\delta}$ in the normal space $X$, there is a continuous $u: X \rightarrow[0,1]$ such that $A=u^{-1}(0)$. Let $\varrho$ be a compatible metric on $Y$, and let

$$
V=\{(x, y) \in(X \backslash A) \times Y: \varrho(y, f(x))<u(x)\} .
$$

Then $V$ is open in $(X \backslash A) \times Y$ and $\varphi(x) \cap V(x) \neq \emptyset$ for all $x \in X \backslash A$. Define $\alpha: X \backslash A \rightarrow 2^{Y}$ by $\alpha(x)=\varphi(x) \cap V(x)$. Then $\alpha$ has the pointwise SNEP by Lemmas 2.1 and 2.2 .

Since $X \backslash A$ is an $F_{\sigma}$ in $X$, we have $X \backslash A$ paracompact (by $\left[\mathrm{M}_{1}\right.$, Proposition 3]) and $\operatorname{dim} X \backslash A=0$. Hence $\alpha$ has a selection $k$ by Case 1 of Lemma 2.3 (with $X$ replaced by $X \backslash A$ and $\varphi$ by $\alpha$ ). Define $h: X \rightarrow Y$ by $h \mid A=g$ and $h \mid(X \backslash A)=k$. This $h$ satisfies all our requirements.

The following corollary should be compared to Lemma 2.3.

Corollary 2.6. Let $X$ be paracompact, $A \subset X$ a closed $G_{\delta}$ with $\operatorname{dim}_{X} X \backslash A=0$, and let $\varphi: X \rightarrow 2^{Y}$ be l.s.c. and have the SNEP at every $x \in X \backslash A$. Then $\varphi$ has the SEP at A. More generally, every selection $g$ for $\bar{\varphi} \mid A$ extends to a continuous $k: X \rightarrow Y$ such that $k(x) \in \varphi(x)$ for every $x \in X \backslash A$.

Proof. We may clearly suppose that $Y$ is completely metrizable. Since $\bar{\varphi}$ is also l.s.c., $g$ extends to a selection $f$ for $\bar{\varphi}$ by Theorem 1.2. Hence, by Lemma 2.5, $g$ extends to a continuous $k: X \rightarrow Y$ such that $k(x) \in \varphi(x)$ for every $x \in X \backslash A$.

3. Proof of Theorem 1.4. Let $g$ be a selection for $\varphi \mid A$. We must extend $g$ to a selection $f$ for $\varphi$. 
Let $B_{0}=A$ and let $B_{n}=A \cup \bigcup_{i=1}^{n} C_{i}$ for $n>0$. Let $\varrho$ be a compatible, complete metric on $Y$. By induction, we will construct selections $f_{n}$ for $\bar{\varphi}$ such that:
(a) $f_{0} \mid A=g$,
(b) $f_{n+1}(x)=f_{n}(x) \in \varphi(x)$ for $x \in B_{n}$,
(c) $\varrho\left(f_{n+1}(x), f_{n}(x)\right) \leq 2^{-n}$ for all $x \in X$.

Suppose that we had such functions $f_{n}$. Then $\left(f_{n}\right)$ converges uniformly to a selection $f$ for $\bar{\varphi}$ by (c). Clearly $f$ extends $g$ by (a) and (b). Also $f(x) \in \varphi(x)$ for $x \in \bigcup_{n} B_{n}$ by (b), and $f(x) \in \varphi(x)$ for $x \notin \bigcup_{n} B_{n}$ because $\varphi(x)=\bar{\varphi}(x)$ for such $x$ by our hypotheses. Hence $f$ is a selection for $\varphi$ which extends $g$.

It remains to construct the sequence $\left(f_{n}\right)$. Since $\varphi$ is l.s.c., so is $\bar{\varphi}$, and hence $g$ extends to a selection $f_{0}$ for $\bar{\varphi}$ by Theorem 1.2. Now suppose $f_{0}, \ldots, f_{n}$ have been chosen, and let us choose $f_{n+1}$. Let

$$
W=\left\{(x, y) \in X \times Y: \varrho\left(y, f_{n}(x)\right)<2^{-n}\right\}
$$

Then $W$ is open in $X \times Y$ and $\varphi(x) \cap W(x) \neq \emptyset$ for all $x \in X$. Define $\alpha: X \rightarrow$ $2^{Y}$ by $\alpha(x)=\varphi(x) \cap W(x)$. Since $\varphi \mid C_{n+1}$ has the SNEP, so does $\alpha \mid C_{n+1}$ by Lemma 2.2. Also $\operatorname{dim}_{C_{n+1}} C_{n+1} \backslash\left(C_{n+1} \cap B_{n}\right) \leq 0$ because $\operatorname{dim}_{X} X \backslash A=0$ and $B_{n} \supset A$. Hence, by Lemma 2.3, the selection $f_{n} \mid\left(C_{n+1} \cap B_{n}\right)$ for $\alpha \mid\left(C_{n+1} \cap B_{n}\right)$ extends to a selection $h$ for $\alpha \mid C_{n+1}$. Define $k: B_{n+1} \rightarrow Y$ by $k\left|B_{n}=f_{n}\right| B_{n}$ and $k \mid C_{n+1}=h$. This $k$ is a selection for $\alpha \mid B_{n+1}$. Now $\alpha: X \rightarrow 2^{Y}$ is l.s.c. by Lemma 2.2 , and hence so is $\bar{\alpha}: X \rightarrow \mathcal{F}(Y)$. Since $\operatorname{dim}_{X} X \backslash B_{n+1} \leq 0$ (because $B_{n+1} \supset A$ ), $k$ extends to a selection $f_{n+1}$ for $\bar{\alpha}$ by Theorem 1.2. This $f_{n+1}$ has all required properties.

4. Proof of Theorem 1.5. The proof proceeds along the same lines as the proof of Theorem 1.4, but the details are more complicated.

Let $g$ be a selection for $\varphi \mid A$. We must extend $g$ to a selection $f$ for $\varphi$.

Let $B_{0}=A$ and let $B_{n}=A \cup \bigcup_{i=1}^{n} C_{i}$ for $n>0$. Then $B_{n}$ is a $G_{\delta}$ in $X$ for all $n$. Let $\varrho$ be a compatible, complete metric on $Y$. Since $Z$ is a $G_{\delta}$ in $Y$, it is completely metrizable; let $d$ be a compatible, complete metric on $Z$. Finally, define $\psi: X \rightarrow \mathcal{F}(Z)$ by $\psi(x)=\bar{\varphi}(x) \cap Z$. Our assumptions imply that $\psi(x)=\varphi(x)$ for $x \notin C$ and that $\bar{\psi}(x)=\bar{\varphi}(x)$ for all $x \in X$. Thus $\bar{\psi}$ is l.s.c., and hence so is $\psi$.

By induction, we will construct selections $f_{n}$ for $\bar{\varphi}$ such that:
(a) $f_{0} \mid A=g$,
(b) $f_{n+1}(x)=f_{n}(x) \in \varphi(x)$ for $x \in B_{n}$,
(c) $\varrho\left(f_{n+1}(x), f_{n}(x)\right)<2^{-n}$ for all $x \in X$,
(d) $f_{n}(x) \in \psi(x)$ for $x \notin B_{n}$,
(e) $d\left(f_{n+1}(x), f_{n}(x)\right)<2^{-n}$ for $x \notin B_{n+1}$. 
Suppose that we had such functions $f_{n}$. Then $\left(f_{n}\right)$ converges $\varrho$-uniformly to a continuous $f: X \rightarrow Y$ by (c), and $f$ extends $g$ by (a) and (b). Finally, if $x \notin \bigcup_{n} B_{n}$ then $f(x) \in \psi(x)=\varphi(x)$ by (d) and (e), and if $x \in \bigcup_{n} B_{n}$ then $f(x) \in \varphi(x)$ by (a) and (b). Thus $f$ is a selection for $\varphi$ which extends $g$.

It remains to construct the sequence $\left(f_{n}\right)$. Since $\bar{\varphi}$ is l.s.c., $g$ extends to a selection $g^{\prime}$ for $\bar{\varphi}=\bar{\psi}$ by Theorem 1.2 . Also $X \backslash A$ is an $F_{\sigma}$ in $X$, so $X \backslash A$ is paracompact (by $\left[\mathrm{M}_{1}\right.$, Proposition 3]) and $\operatorname{dim} X \backslash A=0$. Hence $\psi \mid(X \backslash A): X \backslash A \rightarrow \mathcal{F}(Z)$ has the SEP by Theorem 1.2. By Lemma $2.5, g$ therefore extends to a continuous $f_{0}: X \rightarrow Y$ such that $f_{0}(x) \in \psi(x)$ for $x \in X \backslash A$.

Suppose now that we have $f_{0}, \ldots, f_{n}$. To construct $f_{n+1}$, we will first extend $f_{n} \mid B_{n}$ to a suitable selection $h$ for $\varphi \mid B_{n+1}$ and will then extend $h$ to obtain $f_{n+1}$.

Since $f_{n}(x) \in \psi(x) \subset Z$ for all $x \in X \backslash B_{n}$, we can define

$$
V=\left\{(x, y) \in\left(X \backslash B_{n}\right) \times Z: d\left(y, f_{n}(x)\right)<2^{-n}\right\} .
$$

Then $V$ is relatively open in $X \times Z$, so $V=\widetilde{V} \cap(X \times Z)$ for some open $\widetilde{V}$ in $X \times Y$. Define

$$
W=\widetilde{V} \cap\left\{(x, y) \in X \times Y: \varrho\left(y, f_{n}(x)\right)<2^{-n}\right\},
$$

so $W$ is open in $X \times Y$.

For all $x \in X \backslash B_{n}$ we have $f_{n}(x) \in \psi(x) \subset \bar{\varphi}(x)$ and $f_{n}(x) \in W(x)$; we can thus define $\alpha: X \backslash B_{n} \rightarrow 2^{Y}$ by $\alpha(x)=\varphi(x) \cap W(x)$, and we note that $f_{n}(x) \in \bar{\alpha}(x)$ for all $x \in X \backslash B_{n}$. Since $\varphi \mid C_{n+1}$ has the pointwise SNEP, so does $\varphi \mid\left(B_{n+1} \backslash B_{n}\right)$ by Lemma 2.1 , and hence so does $\alpha \mid\left(B_{n+1} \backslash B_{n}\right)$ by Lemma 2.2. Also $B_{n}$ is a $G_{\delta}$ in $X$, and $\operatorname{dim}_{B_{n+1}} B_{n+1} \backslash B_{n} \leq 0$ because $\operatorname{dim}_{X} X \backslash A=0$ and $B_{n} \supset A$. Thus, by Lemma 2.5, $f_{n} \mid B_{n}$ extends to a continuous $h: B_{n+1} \rightarrow Y$ such that $h(x) \in \alpha(x)$ for every $x \in B_{n+1} \backslash B_{n}$.

Define $\beta: X \rightarrow 2^{Y}$ by

$$
\beta(x)= \begin{cases}\left\{f_{n}(x)\right\} & \text { if } x \in B_{n}, \\ \psi(x) \cap W(x) & \text { if } x \in X \backslash B_{n} .\end{cases}
$$

Using Corollary 2.6, we will extend $h$ to a continuous $f_{n+1}: X \rightarrow Y$ such that $f_{n+1}(x) \in \beta(x)$ for every $x \in X \backslash B_{n+1}$.

To apply Corollary 2.6, recall that $B_{n+1}$ is a $G_{\delta}$ in $X$, and note that $\beta$ is l.s.c. because $\beta \mid\left(X \backslash B_{n+1}\right)$ is l.s.c. (by Lemma 2.2) and $f_{n}(x) \in \beta(x)$ for all $x \in X$. Next, every closed (in $X$ ) $E \subset X \backslash A$ is paracompact and 0-dimensional, so $\psi \mid E: E \rightarrow \mathcal{F}(Z)$ has the SEP by Theorem 1.2; it follows that $\psi$ has the SNEP at every $x \in X \backslash A$, and hence so does $\beta$. Finally, $h$ is a selection for $\bar{\beta} \mid B_{n+1}$, because if $x \in B_{n}$ then $h(x)=f_{n}(x) \in \beta(x)$, and if $x \in B_{n+1} \backslash B_{n}$ then $h(x) \in \varphi(x) \cap W(x) \subset \bar{\psi}(x) \cap W(x) \subset \bar{\beta}(x)$. By Corollary 2.6, we can therefore extend $h$ to a continuous $f_{n+1}: X \rightarrow Y$ such 
that $f_{n+1}(x) \in \beta(x)$ for every $x \in X \backslash B_{n+1}$, and this $f_{n+1}$ satisfies all our requirements.

5. Proof of Theorem 1.7. The proof of Theorem 1.7 is similar to the proof of Theorem 1.5, with two principal modifications. The first of these, which is easy, replaces references to Theorem 1.2 by references to the following generalization of Theorem 1.2.

TheOREM $5.1\left[\mathrm{M}_{7}\right.$, Theorem 1.2(b)]. Let $X, Y$ and $A \subset X$ be as in Theorem 1.2, let $Z \subset X \times Y$ be $a G_{\delta}$, and let $\varphi: X \rightarrow 2^{Y}$ be l.s.c. with $\varphi(x) \in \mathcal{F}(Z(x))$ for every $x \in X$. Then $\varphi$ has the $S E P$ at $A$.

The second modification in the proof of Theorem 1.5 is more technical, and concerns condition (e) on the sequence $\left(f_{n}\right)$. That condition depends on the existence of a compatible, complete metric $d$ on $Z \subset Y$, and no analogue of such a metric appears to be available for $Z \subset X \times Y$ in Theorem 1.7. We now indicate how that difficulty can be circumvented.

Since Theorem 1.7 assumes that $Z$ is a $G_{\delta}$-subset of $X \times Y$, we can write $Z=\bigcap_{n} G_{n}$ with each $G_{n}$ open in $X \times Y$. Now inductively construct two sequences, a sequence $\left(f_{n}\right)$ of selections for $\bar{\varphi}$ and a sequence $\left(H_{n}\right)$ of open subsets of $X \times Y$, satisfying conditions (a)-(d) for $\left(f_{n}\right)$ in the proof of Theorem 1.5 as well as the following replacement for condition (e):

$$
\left(\mathrm{e}^{\prime}\right) f_{n+1}(x) \in H_{n+1}(x) \subset \overline{H_{n+1}(x)} \subset H_{n}(x) \cap G_{n+1} \text { for } x \notin B_{n} .
$$

This guarantees that $f=\lim _{n} f_{n}$ satisfies all our requirements.

To construct the sequences $\left(f_{n}\right)$ and $\left(H_{n}\right)$, we begin by setting $G_{0}=$ $H_{0}=X \times Y$ and choosing $f_{0}$ as in the proof of Theorem 1.5 (but invoking Theorem 5.1 instead of Theorem 1.2). Once we have $f_{0}, \ldots, f_{n}$ and $H_{0}, \ldots, H_{n}$, we note that $f_{n}(x) \in H_{n}(x) \cap G_{n+1}$ for $x \notin B_{n}$, and we choose an open $H_{n+1}$ in $\left(X \backslash B_{n}\right) \times Y$ such that

$$
f_{n}(x) \in H_{n+1}(x) \subset \overline{H_{n+1}(x)} \subset H_{n}(x) \cap G_{n+1} \quad \text { for } x \notin B_{n} .
$$

(The existence of such an $H_{n+1}$ follows easily from the fact that $X \backslash B_{n}$, being an $F_{\sigma}$ in $X$, is paracompact.) Having chosen $H_{n+1}$, we now construct $f_{n+1}$ as in the proof of Theorem 1.5 , with $\widetilde{V}$ replaced by $H_{n+1}$ in the definition of $W$ and with the reference to Theorem 1.2 replaced by a reference to Theorem 5.1.

6. Examples. Our first example shows that the requirement in Theorem 1.5 that the $C_{n}$ 's are all $G_{\delta}$-subsets of $X$ cannot be omitted. As usual, $\mathbb{R}$ denotes the reals, $\mathbb{Q}$ the rationals, and $\mathbb{P}$ the irrationals.

EXAMPLE 6.1. Let $X$ be the one-point compactification of an uncountable discrete space, and let $x^{*} \in X$ be the point at infinity. Let $Y=\mathbb{R}$, 
and define $\varphi: X \rightarrow 2^{Y}$ by $\varphi\left(x^{*}\right)=\mathbb{Q}$ and $\varphi(x)=\mathbb{P}$ if $x \neq x^{*}$. Then all assumptions of Theorem 1.5 are satisfied, with $C_{1}=\left\{x^{*}\right\}$ and with $A$ and the other $C_{n}$ 's all empty, except that $C_{1}$ is not a $G_{\delta}$ in $X$. Nevertheless, $\varphi$ has no selection.

Proof. Suppose $f$ were a selection for $\varphi$, and let $y^{*}=f\left(x^{*}\right)$. Then $\left\{x^{*}\right\}=f^{-1}\left(y^{*}\right)$, so $\left\{x^{*}\right\}$ is a $G_{\delta}$ in $X$, which is impossible.

Our next example shows that the requirement in Theorem 1.5 that $A$ is a $G_{\delta}$ in $X$ cannot be omitted.

ExAMPLE 6.2. Let $X$ be a first-countable, paracompact space with $\operatorname{dim} X=0$ which has a countable, closed, non- $G_{\delta}$ subset $A=\left\{x_{1}, x_{2}, \ldots\right\}\left(^{5}\right)$. Let $Y=\mathbb{R}$, and define $\varphi: X \rightarrow 2^{Y}$ by $\varphi(x)=\mathbb{Q}$ if $x \in A$ and $\varphi(x)=\mathbb{P}$ if $x \notin A$. Then all assumptions of Theorem 1.5 are satisfied with $C_{n}=\left\{x_{n}\right\}$, except that $A$ is not a $G_{\delta}$ in $X$. However, $\varphi$ does not have the SEP at $A$.

Proof. Let $g$ be the selection for $\varphi \mid A$ defined by $g(x)=0$ for $x \in A$. If $g$ extended to a selection $f$ for $\varphi$, then $A=f^{-1}(0)$ would be a $G_{\delta}$ in $X$, a contradiction.

Our last example, which was kindly provided by Roman Pol, shows that Theorem 1.5 becomes false if the requirement that $\bar{\varphi}(x) \cap Z$ is dense in $\bar{\varphi}(x)$ for $x \in C$ is omitted.

EXAMPLE 6.3. Let $X$ be a non-empty, completely metrizable space without isolated points, let $Y=[0,1]$ and let $Z=(0,1]$. Then there exists a countable $C \subset X$, and a l.s.c. $\varphi: X \rightarrow 2^{Y}$ with $\varphi(x)=Z$ for $x \notin C$ and with $\varphi(x) \in \mathcal{F}(Y)$ for $x \in C$, such that $\varphi$ has no selection.

Proof. Let $x_{0} \in X$. Since no $x \in X$ has a countable neighborhood, we can choose disjoint countable sets $C_{0}, C_{1}, \ldots$ in $X$, with $C_{0}=\left\{x_{0}\right\}$, such that each $C_{0} \cup \ldots \cup C_{n}$ is closed in $X$ and is the set of accumulation points of $C_{n+1}$. Let $C=C_{0} \cup C_{1} \cup \ldots$, and define $\varphi: X \rightarrow 2^{Y}$ by

$$
\varphi(x)= \begin{cases}\{0\} & \text { if } x=x_{0}, \\ \{0\} \cup[1 / n, 1] & \text { if } x \in C_{n}, \\ (0,1] & \text { if } x \notin C .\end{cases}
$$

It is easily checked that $\varphi$ is l.s.c., so it remains to show that $\varphi$ has no selection.

Suppose $f$ were a selection for $\varphi$. Let $D=f^{-1}(0)$. Then $x_{0} \in D \subset C$ and $D$ is closed in $X$. Also $D$ has no isolated points, for if $x \in D \cap C_{n}$, there is a sequence $x_{i} \rightarrow x$ with $x_{i} \in C_{n+1}$; since $f(x)=0$ and $f\left(x_{i}\right) \in$

$\left({ }^{5}\right)$ One can, for example, take $X$ to be the set $\mathbb{R}$, topologized by $\{U \cup S: U$ open in $\mathbb{R}$, $S \subset \mathbb{P}\}$ (this $X$ is sometimes called the Michael line), and take $A$ to be the rationals in $X$. See $\left[\mathrm{M}_{5}\right]$ or $[\mathrm{B}, \mathrm{pp} .390-391]$. 
$\{0\} \cup[1 /(n+1), 1]$ for all $i$, it follows that $f\left(x_{i}\right)=0$ (and hence $x \in D$ ) for almost all $i$. Thus $D$ is a non-empty, closed, countable subset of $X$ without isolated points, and that is impossible.

\section{References}

[B] D. Burke, Covering properties, in: Handbook of Set-Theoretic Topology, NorthHolland, Amsterdam 1984, 347-422.

$\left[\mathrm{M}_{1}\right]$ E. Michael, A note on paracompact spaces, Proc. Amer. Math. Soc. 4 (1953), 831-838.

$\left[\mathrm{M}_{2}\right] \quad$ - Selected selection theorems, Amer. Math. Monthly 63 (1956), 233-238.

$\left[\mathrm{M}_{3}\right] \quad-$, Continuous selections I, Ann. of Math. 63 (1956), 361-382.

$\left[\mathrm{M}_{4}\right] \quad-$, Continuous selections II, ibid. 64 (1956), 562-580.

$\left[\mathrm{M}_{5}\right]$ - , The product of a normal space and a metric space need not be normal, Bull. Amer. Math. Soc. 69 (1963), 375-376.

$\left[\mathrm{M}_{6}\right]-$, Continuous selections and countable sets, Fund. Math. 111 (1981), 1-10.

$\left[\mathrm{M}_{7}\right]-$, A generalization of a theorem on continuous selections, Proc. Amer. Math. Soc. 105 (1989), 236-243.

DEPARTMENT OF MATHEMATICS

UNIVERSITY OF WASHINGTON

SEATTLE, WASHINGTON 98195

U.S.A. 